\title{
Implementation of Real Time Graphical User Interface Library for Dynamic Reconfigurable System
}

\author{
Vaibhawa Mishra \\ CSIR-CEERI \\ Digital Systems Group \\ Pilani, Rajasthan, India-333031
}

\author{
Kota Solomon Raju \\ CSIR-CEERI \\ Digital Systems Group \\ Pilani, Rajasthan, India-333031
}

\author{
Pramod Tanwar \\ CSIR-CEERI \\ Digital Systems Group \\ Pilani, Rajasthan, India-333031
}

\begin{abstract}
This work presents the implementation of real time GUI library for FPGA based run time reconfigurable system that is targeted to ML507 board. The presented work implements floating point arithmetic core as a reconfigurable IP and operands can be provide to the above core with GUI running on display monitor. User can interact with GUI with keyboard and mouse. GUI allows user to change the functionality of the circuit in run time by clicking on appropriate buttons. This approach combines both HW and SW flows. The HW module can be reconfigured on the fly by using partial dynamic reconfiguration.
\end{abstract}

\section{Keywords}

Partial Reconfiguration, Real Time Processing, GUI, floating point arithmetic unit.

\section{INTRODUCTION}

In computing, GUI allows users to interact with the system with display instead of text commands. Windows and menus can be easily manipulated by a mouse. Now, a GUI based application is not implemented to only high computing machines but they can be easily ported to small devices like FPGA based embedded system [1]. These FPGA platforms provide a flexible way to implement a complex design and facilitate us to change the sub set of design in run time that allow reutilizing currently unused part of the circuit. These features of the FPGA are being used widely in signal processing, image processing, and complex systems [2]. Reconfiguration feature can also be used in operating system environment [3].

In this paper, we present self-reconfigurable architecture with real time GUI library support. In particular, we propose a floating point unit architecture which dynamically alternates itself on demand by reconfiguring a portion of the logic. The floating point unit contains single precision floating point adder, multiplier, subtracter and divider. The operands as well as reconfigurable command sends through reconfiguration control panel.

This paper is organized as follows. Section 2 presents brief description of proposed hardware architecture. Architecture analysis of GUI library has been presented in Section 3. Method for user interaction is given Section 4. The details of the reconfiguration controller have been discussed in Section 5. Section 6 will provide analysis of implementation. At last, results have been presented in Section 7 .

\section{PROPOSED HARDWARE ARCHITECTURE}

Our proposed hardware design is based on PowerPC440 hard core processor. Our design is composed of one static region and one floating point reconfigurable arithmetic region. The Hard core PowerPC is used to run GUI for reconfiguration.
All arithmetic modules are implemented as four individual modules and can be swapped in and out on demand using reconfiguration method.

As shown in Fig. 1, the processor is connected to Processor Local Bus together with external DDR2 memory, the timer peripheral, the UART controller, Compact FLASH controller, DVI controller and PS/2 controller. The Compact FLASH is used to store the partial bit files of floating point adder, multiplier, divider and subtractor for reconfiguration. We are using dual ported DDR2 memory whose one port is connected to DVI controller through Xilinx NPI interface and other port is connected to PPC440 memory controller. PS/2 controller has been attached to the system to respond mouse and keyboard activity. Our design also incorporates HwIcap IP that enables processor to reconfigure the device at run time.

We have followed the IEEE 75432 - bit single precision data format to represent floating point numbers. All floating point modules are designed in such a way that it takes operands in 32 - bit hexadecimal formats and produce the output in same pattern. The details of the algorithm used to design the floating point arithmetic module are beyond the scope of this paper and adopted as in [4].

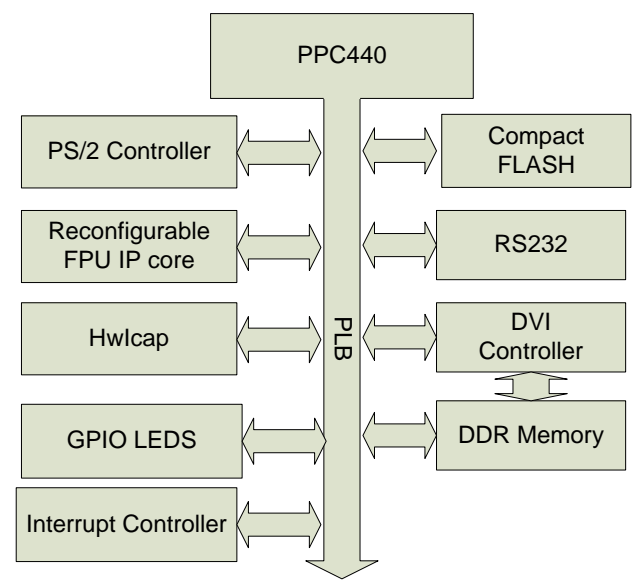

Fig 1 Schematic of proposed reconfigurable architecture

\section{SOFTWARE ARCHITECTURE}

Our proposed software architecture can be implemented in two parts; the $1^{\text {st }}$ part is implementation of GUI library and $2^{\text {nd }}$ part is implementation of GUI based application. We have implemented reconfiguration controller as a GUI application. The GUI library basically manages the hardware resources such as display monitor, input devices, memory and timer. These libraries are designed in such a way that it can manage all the requirements of every running GUI application. To answer the request from all the application currently running on the system, this library should be as real time process that 
schedules and executes various types of tasks. To update the display monitor continuously, the GUI library should know the number of running GUI application and opened GUI windows so that it can send command to each application or window to reproduce their pixels. In return, each GUI application listen the request by involving a set of graphical entity having pixel representation of the GUI windows. Consequently, the time required to update the screen depends on number and type of graphical entity selected by each running GUI application's pixels.

\section{USER INTERACTION}

In our implementation, user can interact with the running application with keyboard and mouse both attached with PS/2 controllers IP at sample rate of $16 \mathrm{~K}$ bits per second. Mouse generates a stream of motion during its movements. Each motion or keyboard input generates interrupt to the GUI library. Each motion coordinate need to be converted into display coordinates. This also includes movement of mouse cursor, changing the GUI window element or its position. For input from the keyboard the scan code need to be converted to its related ASCII and stand in buffer. We have used slider window to provide operands to the system. In this step, a scale of values is displayed on the monitor for both operands. User has to slide the scale and then to click on respected buttons to pass the both operands to floating point unit. The range of the scale for the both operands is from 0 to 255 in step of 0.0001 . The result will be displayed after pressing the result button.

\section{RECONFIGURATION CONTOLLER}

Reconfiguration controller completely written in $\mathrm{C}$ with driver function provided by Xilinx $\mathrm{C}$ libraries. Each reconfiguration task as well as data execution task is bind with buttons appeared on the monitor. Each module replacement task is bind with individual buttons. Only one of all four buttons will be active at once. Reconfiguration controller performs the following action. First, it will identify the partial bit files related to the module and secondly, it will reconfigure the portion of the FPGA using that bit stream.

The ICAP API has been used for transferring the bit file data between the external configuration memory and PLB BRAM, working as configuration cache. The ICAP API provides method for accessing FPGA resources through ICAP port. Before using the HwIcap module, one must initialize it..

\section{IMPLEMENTATION ANALYSIS}

The idea is implemented and tested on ML507 board having xc5vfx70t-1ff1136 device of Xilinx Virtex family. The complete set-up is as shown in Fig. 2.

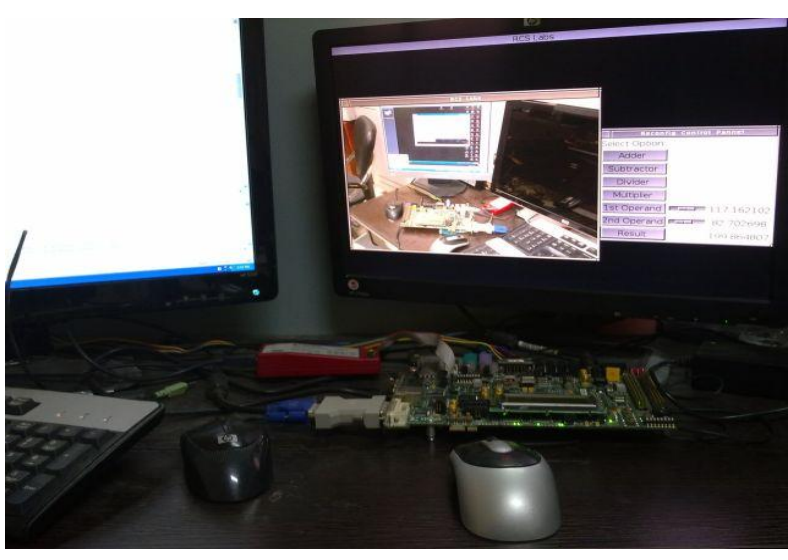

Fig 2Experimental Setup of the System

Since our approach is HW/SW co-design, the base system is designed with Xilinx Platform Studio tools. The GUI library has been compiled using EDK shell using powerpc-eabi compilers for PowerPC processor [5]. Partial Reconfiguration flow has been adapted using EAPR [6].The GUI library is written in C. PowerPC has been clocked on $300 \mathrm{MHz}$. Rest of the IP cores are running at PLB Bus Frequency that is 100 MHz. Only VGA controller IP core is running at $50 \mathrm{MHz}$. the VGA controller uses PLB for accessing the control registers and NPI interface of multi-port memory controller for fetching data from frame buffer.

To get interrupt of the PS/2 controller, we have connected the $\mathrm{PS} / 2$ interrupt line to the interrupt line of "xps_intc" peripheral. The "IRQ" port of the peripheral "xps_intc" is then connected to port "EICC440EXTIRQ" of the PPC440 processor.

\section{RESULTS}

System was designed with minimum number of peripherals. We have successfully implemented and tested our proposed architecture. Table 1 provides the device utilization summary of all individual modules. The given resource utilization is only for the PRMs used in the design. As it has been discussed earlier, floating point arithmetic unit has been taken as reconfiguration module. The simulation results for floating point addition, multiplication, subtraction and division are shown in the Fig. 3, 4, 5 and 6 respectively.

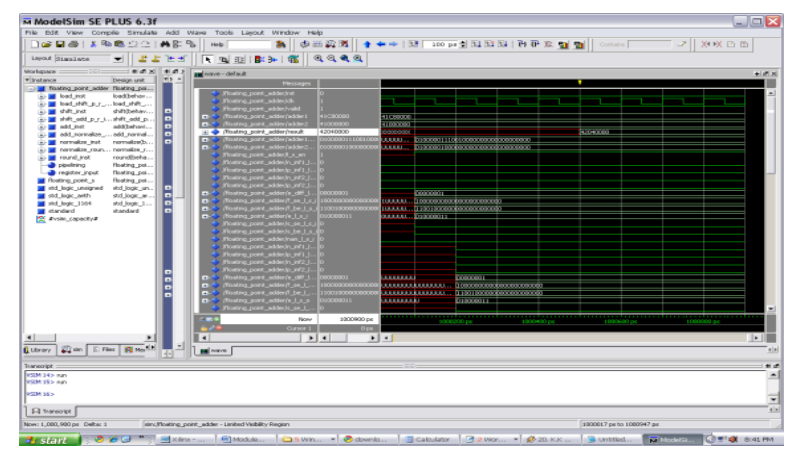

Fig 3 Simulation result of floating point adder 


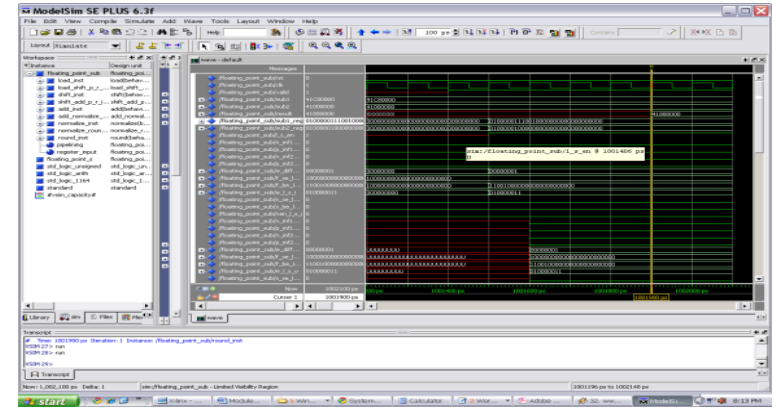

Fig. 4 Simulation result of floating point subtractor

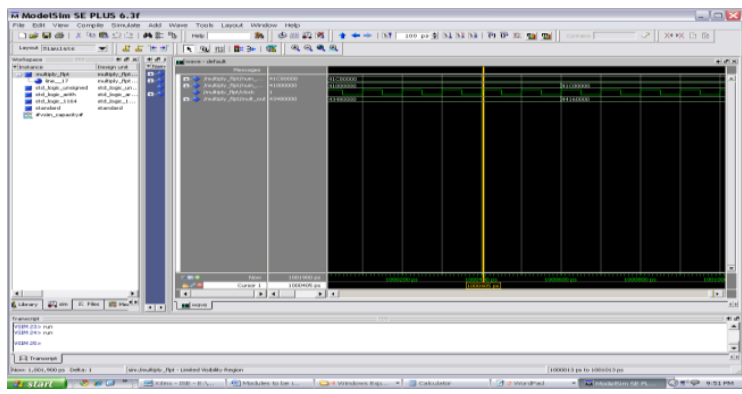

Fig. 5 Simulation result of floating point multiplier

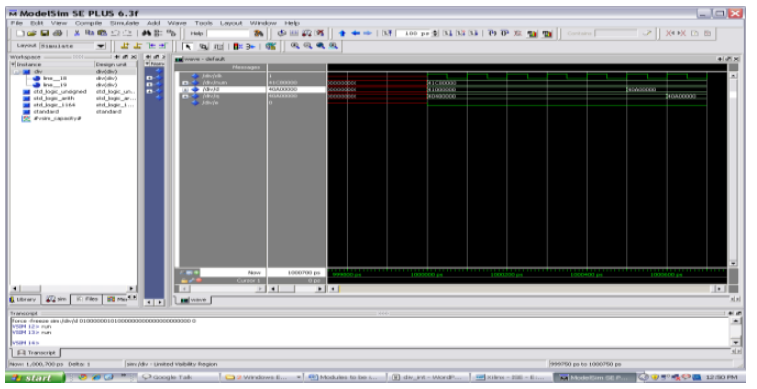

Fig. 6 Simulation result of floating point divider

When the system boots, an initial bit file to invoke all the hardware is required. The FPGA is configured with Xilinx impact tool and once system is ready, the executable is transferred via XMD. When the system completes its booting, reconfiguration application is selected and displayed on the monitor. Fig 7 shows the output when adder has been selected and Fig. 8 Shows when adder has been completely replaced by multiplier module in reconfigurable region.

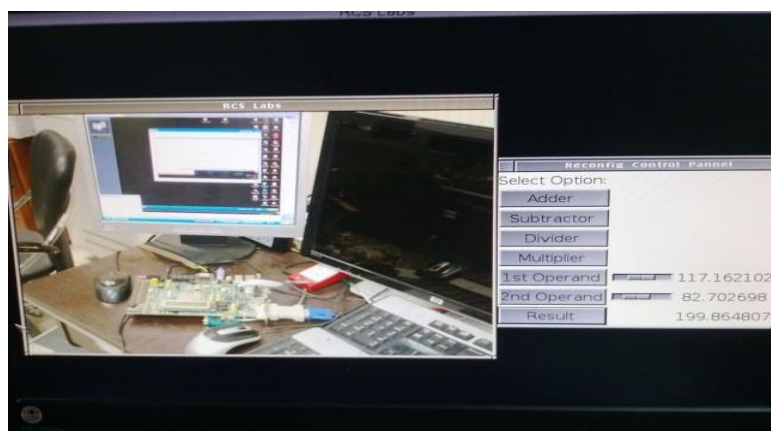

Fig. 7 Control panel result when floating point adder is as PRM

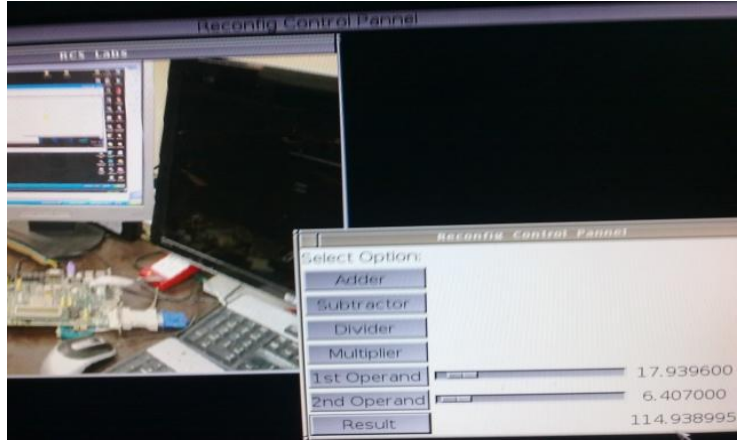

Fig. 8 Control panel result when floating point multiplier is as PRM

Table 1 Resource usage of PRMs for Virtex 5 FXT

\begin{tabular}{|c|c|c|c|c|c|c|c|c|c|}
\hline \multirow{3}{*}{\multicolumn{2}{|c|}{$\begin{array}{c}\text { Floating Point } \\
\text { Arithmetic } \\
\text { Modules }\end{array}$}} & \multicolumn{8}{|c|}{ Resource Used } \\
\hline & & \multicolumn{2}{|c|}{ Slice LUTs } & \multicolumn{2}{|c|}{$\begin{array}{l}\text { Full Used } \\
\text { LUT FF } \\
\text { pairs }\end{array}$} & \multicolumn{2}{|c|}{$\begin{array}{c}\text { Slice } \\
\text { Registers }\end{array}$} & \multicolumn{2}{|c|}{ DSP48Es } \\
\hline & & Min & $\operatorname{Max}$ & Min & Max & Min & Max & Min & Max \\
\hline \multirow{4}{*}{ PRR1 } & Adder & 955 & 44800 & 251 & 1183 & 479 & 44800 & - & 128 \\
\hline & Multiplier & 128 & 44800 & 93 & 247 & 212 & 44800 & 2 & 128 \\
\hline & Divider & 2357 & 44800 & 93 & 2565 & 301 & 44800 & 2 & 128 \\
\hline & Subtractor & 956 & 44800 & 175 & 1183 & 480 & 44800 & - & 128 \\
\hline
\end{tabular}

\section{REFERENCES}

[1] www.genode-labs.com/products/fpga-graphics.

[2] Vaibhawa Mishra, Kota Solomon Raju and Pramod Tanwar. "Implementation of Dynamic Reconfigurable Audio Player and Spectrum Analyzer". International Journal of Computer Applications 54(9):8-11, September 2012

[3] Vaibhawa Mishra, Kota Solomon Raju, Pramod Tanwar, "Implantation of Dynamically Reconfigurable Systems on Chip with OS Support", International Journal of Computer Applications (IJCA), Vol. 49, No 6, July, 2012, pp. 33-35

[4] http://www.ece.uvic.ca/ elec499/2004a/group05/html/ math.html

[5] Xilinx Inc., "Embedded System Tools Reference Manual," Version 3.0, 2004.

[6] Xilinx, Inc., "PlanAhead Software Tutorials: Partial Reconfiguration of a processor Peripheral", User Guide UG744, Version 12.3, September 21, 2010 